\title{
Olaratumab: a platelet-derived growth factor receptor- $\alpha$-blocking antibody for the treatment of soft tissue sarcoma
}

This article was published in the following Dove Press journal:

Clinical Pharmacology:Advances and Applications

\section{Alexandra Pender ${ }^{1}$ \\ Robin L Jones ${ }^{1,2}$}

'Sarcoma Unit, Department of Medicine, The Royal Marsden NHS

Foundation Trust, ${ }^{2}$ Division of Clinical

Studies, The Institute of Cancer

Research, London, UK
Correspondence: Robin L Jones Sarcoma Unit, The Royal Marsden Hospital, Fulham Road, London SW3 6JJ, UK

Tel +442078082137

Fax +442078082113

Email robin.jones4@nhs.net

\begin{abstract}
The outcome of patients with unresectable or metastatic soft tissue sarcoma (STS) remains poor with few treatment options. A number of randomized trials in the first-line setting have shown no difference in overall survival between combination anthracycline schedules and single-agent doxorubicin. A Phase Ib/randomized Phase II trial of doxorubicin with or without the platelet-derived growth factor receptor- $\alpha$ (PDGFR $\alpha$ )-blocking antibody, olaratumab, demonstrated a significant difference in median overall survival in favor of the olaratumab arm. The results of this trial led to the approval of olaratumab in combination with doxorubicin in adult anthracycline-naïve unresectable STS. In this review, we discuss the potential role of PDGFR $\alpha$ signaling, early clinical data with olaratumab in sarcomas, the Phase Ib/II trial and ongoing trials with olaratumab in sarcomas.
\end{abstract}

Keywords: olaratumab, sarcoma, PDGFR

\section{Introduction}

Therapeutic options for advanced and metastatic soft tissue sarcomas (STSs) are limited, and median overall survival (OS) is approximately 18 months. The role of doxorubicin as first-line therapy in adult advanced STS is well established. ${ }^{1-3}$ The addition of other agents in combination with doxorubicin has yet to show an additional survival benefit despite encouraging Phase II results, including newer agents such as palifosfamide ${ }^{3}$ and evofosfamide. ${ }^{4}$ Notably, the randomized Phase III trials of both palifosfamide and evofosfamide showed no significant difference in progression-free survival (PFS) or OS as compared with doxorubicin monotherapy. Alternative drug combinations such as gemcitabine and docetaxel have also not shown a PFS or OS benefit. ${ }^{5}$

\section{Platelet-derived growth factor receptor (PDGFR) signaling in cancer}

The potential importance of targeting PDGFR signaling in cancer paved the way for the development of olaratumab. PDGFR tyrosine kinases are overexpressed in a number of tumor types, ${ }^{6-9}$ including sarcomas, prostate, breast, ovarian and lung cancer. ${ }^{10,11}$ Inhibition of the platelet-derived growth factor receptor- $\alpha$ (PDGFR $\alpha$ ) signaling pathway limits tumor growth and metastasis in vitro and in animal models. ${ }^{12,13}$ Amplification of the genomic region encoding PDGFR $\alpha$ in non-small-cell lung cancer (NSCLC) corresponds to overexpression of PDGFR $\alpha$ in vitro, and the activity of this pathway drives cell growth. ${ }^{7}$ Similarly, the overexpression of PDGF isoforms in mice leads to glioma development. ${ }^{14}$ PDGF isoforms (PDGF-AA, PDGF-AB and PDGF-BB) have 
a role in chemokine signaling to the tumor microenvironment and stimulating stromal cells (Figure 1). ${ }^{15}$ PDGFR $\alpha$ expression in breast cancer is associated with more aggressive and HER2-positive and node-positive disease. ${ }^{6}$ A high level of both PDGFR $\alpha$ and PDGF-A within these tumors supports a possible autocrine mechanism for the role of PDGFR signaling in tumor cell growth. PDGF signaling has also been linked with TGF- $\beta$ signaling and epithelial-mesenchymal transition, a key step in tumor metastasis, in prostate, pancreatic and hepatocellular carcinomas. ${ }^{16}$

\section{Early development of olaratumab}

To inhibit binding of various PDGF ligands and activation of PDGFR $\alpha$, a fully human IgGl monoclonal antibody (mAb) was developed. Olaratumab (IMC-3G3) prevents PDGF-AA and PDGF-BB binding, thus reducing receptor autophosphorylation to background levels, ${ }^{17}$ and selectively inhibits downstream PDGFR $\alpha$ signaling in vitro. The high affinity of the antibody for PDGFR $\alpha(40 \mathrm{pM})$ potentially avoids side effects secondary to multi-kinase receptor inhibition. ${ }^{18,19}$

SK-LMS-1, a leiomyosarcoma cell line, responds to PDGF stimulation. ${ }^{17}$ PDGF-AA is expressed in the culture medium when the cells are grown in vitro, supporting an autocrine role for PDGF signaling in these cells. Olaratumab has antitumor activity against a xenograft model of SK-LMS-1 in nude mice at doses between 6 and $20 \mathrm{mg} / \mathrm{kg}$ twice weekly. Tumor volumes are between $35 \%$ and $67 \%$ smaller with olaratumab treatment than in mice treated with control human IgG. ${ }^{17}$ Tumor cell and stromal cell PDGFR $\alpha$ expression can influence response to drugs affecting PDGFR signaling. Mice xenografts of PDGFR $\alpha$-expressing and PDGFR $\alpha$-negative human lung cancer cell lines were treated with the antihuman olaratumab, and IE10, an anti-mouse PDGFR $\alpha$ mAb. Olaratumab inhibited the growth of PDGFR $\alpha$-expressing xenografts. Xenografts with low levels of tumor PDGFR $\alpha$ expression and high levels of PDGF-AA expression were inhibited by $1 \mathrm{E} 10$ targeting the mouse stromal cells. Thus, PDGF signaling in the stroma may influence the response to anti-PDGF therapeutics as much as tumor PDGF signaling. ${ }^{20}$ Targeting the mouse stromal cells in an animal model of prostate cancer did not slow down or prevent the development of skeletal metastases however, whereas olaratumab treatment prevented bone colonization and reduced skeletal disease burden. ${ }^{21}$

\section{Phase I olaratumab data}

A 19-patient Phase I dose-escalation trial evaluated singleagent olaratumab using five different doses and schedule cohorts: 4,8 or $16 \mathrm{mg} / \mathrm{kg}$ weekly or 15 or $20 \mathrm{mg} / \mathrm{kg}$ twoweekly. $^{22}$ The rationale for these doses and schedules was based on observed tumor growth inhibition in U118 glioblastoma and SK-LMS-1 leiomyosarcoma xenograft models at a measured trough concentration of $155-210 \mu \mathrm{g} / \mathrm{mL} .{ }^{17}$ Patients in the weekly cohorts were evaluated after four treatments and 2 weeks of observation (at 6 weeks), and those receiving two-weekly treatments were evaluated after four treatments ( 8 weeks). No dose-limiting toxicities were recorded and so no maximum tolerated dose reached.

The majority of patients enrolled in the study had metastatic prostate cancer $(11 / 19,57.9 \%)$, and no sarcoma patients were enrolled. Eight out of 19 patients (42.1\%) experienced olaratumab-related adverse events, most commonly infusion reactions and fatigue. Two episodes of grade 3 elevated alkaline phosphatase were attributed to olaratumab. Two serious adverse events (grade 2 tumor hemorrhage and grade 2 elevated alkaline phosphatase) were noted as possibly drug related.

The median duration of response was 3.9 weeks $(95 \%$ CI 2.3-8.7 months), and the best response to treatment was

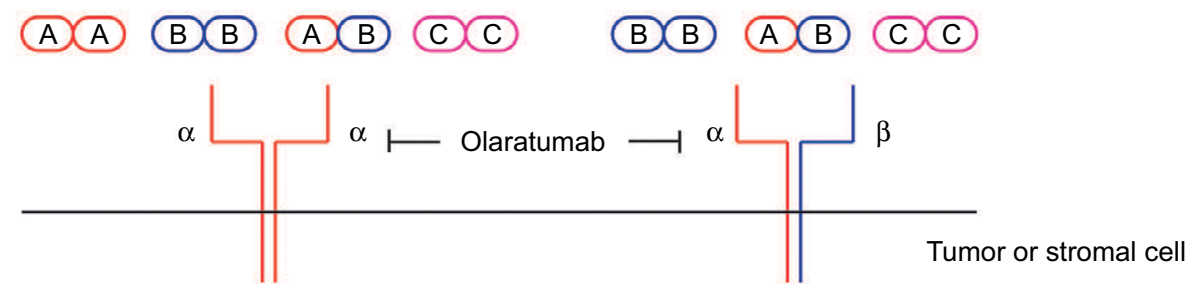

Autocrine tumor growth

Paracrine stromal cell regulation

Angiogenesis

Figure I PDGF ligand signaling through the PDGFR $\alpha$ receptor and the role of olaratumab.

Notes: $\alpha$, PDGFR $\alpha$ receptor; $\beta$, PDGFR $\beta$ receptor; A, PDGF-A isoform; $B$, PDGF-B isoform; C, PDGF-C isoform.

Abbreviations: PDGF, platelet-derived growth factor; PDGFR $\alpha$, PDGF receptor- $\alpha$. 
stable disease. Four (21\%) patients had more than 6 months (range 8.5-13.1 months) of disease stabilization on treatment across a range of dose schedules (4 and $16 \mathrm{mg} / \mathrm{kg}$ weekly and 15 and $20 \mathrm{mg} / \mathrm{kg}$ two-weekly). Pharmocokinetics showed that the $16 \mathrm{mg} / \mathrm{kg}$ weekly and $20 \mathrm{mg} / \mathrm{kg}$ two-weekly schedules resulted in steady state serum trough olaratumab concentrations that were greater than $155 \mu \mathrm{g} / \mathrm{mL}$, the plasma concentration that was effective in xenograft studies, and consequently, these are the recommended dose schedules based on this study.

\section{Olaratumab in sarcomas: Phase Ib/II trial}

The promising activity of olaratumab with doxorubicin in a Phase Ib/II open-label clinical trial ${ }^{23}$ has fueled enthusiasm to take this compound into later-stage clinical trial development. Xenograft models suggest synergism with doxorubicin chemotherapy for up to eight cycles of treatment (unpublished data). In the Phase Ib/II study, olaratumab was given at $15 \mathrm{mg} /$ $\mathrm{kg}$ on day 1 and day 8 of a three-weekly schedule alongside $75 \mathrm{mg} / \mathrm{m}^{2}$ doxorubicin on day 1 every 21 days. ${ }^{23}$ Dexrazoxane was given alongside cycles 5-8 of doxorubicin to reduce the risk of anthracycline cardiotoxicity. ${ }^{24}$

The Phase Ib component of the study recruited 15 patients, and 133 patients were enrolled in the Phase II component. Sixty-six patients were randomized to doxorubicin and olaratumab, and 67 patients to doxorubicin alone in the Phase II study. The proportion of patients with leiomyosarcoma was balanced between the two arms (36.4\% [24/66] doxorubicin and olaratumab vs. $40.3 \%$ [27/67] doxorubicin). The Phase II trial analysis was stratified for histological subtype: leiomyosarcoma, synovial sarcoma and "other" subtypes. Inevitably, as with all trials in STSs, the "other" cohort consisted of a heterogeneous group of subtypes in both arms.

The primary end point of the Phase II study was designed to detect a $50 \%$ improvement in median PFS in the olaratumab and doxorubicin group. This analysis was performed based on investigator assessment of 103 events. Due to the inherent bias of an open-label study, the imaging analysis was retrospectively reviewed in a blinded fashion. The median PFS for olaratumab using investigator assessment was 6.6 months (95\% CI 4.1-8.3) and retrospective review was 8.2 months (95\% CI 5.5-9.8) as compared with 4.1 months (95\% CI 2.8-5.4) and 4.4 months (95\% CI 3.1-7.4), respectively, for doxorubicin alone. To meet the planned criteria for significance, the combination arm would need to extend the PFS interval by approximately 8 weeks, an incremental but potentially meaningful improvement in a patient group, where greater than $50 \%$ had already received one prior line of systemic therapy for local or advanced disease.

Despite the modest increase in PFS with doxorubicin and olaratumab, there was a significant OS benefit observed in the study: 14.7 months (95\% CI 9.2-17.1 months) with doxorubicin alone compared to 26.5 months with the combination (95\% CI 20.9-31.7 months, hazard ratio [HR] 0.46, $p=0.0003$ ). The median exposure of patients in the combination arm to olaratumab was 26.1 weeks (range 3-128 weeks) which extends slightly beyond the time interval required to complete eight sequential doxorubicin treatments three weekly. This may reflect that PDGFR $\alpha$ inhibition affects the tumor microenvironment as much as the tumor cells, and so treatment effects have an impact which continues after cessation of treatment. ${ }^{25}$ Another possibility is through an immune-mediated mechanism that delays the development of further metastatic disease. A discrepancy between PFS and OS has also been seen with a 452-patient study of eribulin vs. dacarbazine in lipo- and leiomyo- sarcomas, ${ }^{26}$ with a PFS of 2.6 months observed in both groups but a median OS of 13.5 months (95\% CI 10.9-15.6) and 11.5 months (95\% CI 9.6-13.0), respectively. These data underline the importance of a preoperative biomarker trial with material-analyzed preand post-olaratumab exposure.

Thirty out of 67 (45\%) patients in the doxorubicin-alone arm went on to receive olaratumab monotherapy post progression for a median of four infusions ( 6 weeks of treatment) as compared with $34 / 66(52 \%)$ of patients in the combination arm who continued olaratumab post combination therapy for a median of nine infusions (13 weeks). This does not suggest a major role for single-agent olaratumab but supports a putative synergism between doxorubicin and olaratumab.

Mean steady state trough olaratumab levels (taken at the end of a cycle between cycles 4 and 9) were measured at $123-156 \mu \mathrm{g} / \mathrm{mL},{ }^{23}$ which approximates to the effective trough level seen in xenograft models. ${ }^{17}$ Interestingly, the patients with the highest quartile average and trough serum olaratumab levels, i.e., greatest exposure, showed a trend toward an improved PFS and OS. ${ }^{23}$

An exploratory analysis of tumor PDGFR $\alpha$ expression did not reveal a significant relationship with PFS or OS; however, the assay was nonspecific and reanalysis of tumors with immunohistochemistry using a more specific antibody identified the expected proportion of detectable PDGFR $\alpha$ expression, which was well balanced between the two arms (33\% patients receiving doxorubicin and olaratumab and $34 \%$ patients receiving doxorubicin). There was no relationship identified between PDGFR $\alpha$ expression and PFS or OS in this trial. 
Grade 3/4 adverse events occurred more frequently in the doxorubicin and olaratumab arm $(38 / 42 \%)$ than the doxorubicinalone $\operatorname{arm}(38 / 31 \%)$, and doxorubicin-associated adverse events were more frequent in the combination arm including neutropenia, nausea and vomiting, diarrhea and mucositis. Thirteen percent of patients in the combination arm had infusion-related reactions. Despite a higher frequency of neutropenia in the doxorubicin and olaratumab group (58 vs. $35 \%$ ), this did not result in more febrile neutropenia or hospital admissions. Treatment discontinuations due to toxicity and deaths were evenly balanced across both arms and nearly all attributable to progressive disease. This is, however, a relatively small patient population with routine use of growth factors on treatment and so larger studies with this combination may give further information about toxicity and mortality. Cardiac complications, excluding peripheral edema, were infrequent and occurred in $8 \%$ of patients treated with doxorubicin and olaratumab and $6 \%$ patients with doxorubicin alone.

In conclusion, this 148-patient Phase Ib/II study in adults with unresectable or metastatic STS reports that the addition of $15 \mathrm{mg} / \mathrm{kg}$ olaratumab on Days 1 and 8 of a 21-day cycle to doxorubicin $75 \mathrm{mg} / \mathrm{m}^{2}$ 3-weekly results in a significant improvement in median OS as compared with doxorubicin alone. The primary end point of the trial was a 50\% improvement in PFS, and the end point was met although the difference in favor of the olaratumab arm was less impressive. This study has led to the Phase III placebo-controlled double-blind ANNOUNCE study of doxorubicin and olaratumab vs. doxorubicin and placebo in advanced or metastatic STS which is expected to report in 2020. In October 2016, the US Food and Drug Administration granted accelerated approval of olaratumab in combination with anthracycline-based chemotherapy in adults with non-curable STS. The European Medicines Agency echoed this decision in November 2016 with "conditional approval" of olaratumab with doxorubicin in the same patient population. ${ }^{27}$

\section{Olaratumab in lung cancer: Phase II trial}

A 131-patient Phase II trial randomized treatment-naïve adult patients with advanced NSCLC to carboplatin, paclitaxel and olaratumab treatment or carboplatin and paclitaxel treatment. ${ }^{28}$
Olaratumab was given at $15 \mathrm{mg} / \mathrm{kg}$ on Days 1 and 8 alongside carboplatin AUC 6 and paclitaxel $200 \mathrm{mg} / \mathrm{m}^{2}$. Up to six cycles of chemotherapy were given and then the patients in the experimental arm received olaratumab maintenance therapy using the same schedule until progression. Patients in the control arm could cross over to the olaratumab arm on progression. There was no significant difference in PFS, OS or response rate between the two arms, and more neutropenia, thrombocytopenia and fatigue was reported in the experimental arm.

\section{Olaratumab in gastrointestinal stromal tumors: Phase II trial}

PDGFRa D842V mutations in gastrointestinal stromal tumors (GIST) confer resistance to imatinib, and this group of patients has poorer outcomes in the metastatic setting. ${ }^{29}$ A small Phase II study evaluated the response to $20 \mathrm{mg} / \mathrm{kg}$ olaratumab every 14 days in adult patients with pretreatment metastatic GIST with and without a PDGFR $\alpha$ D 842 V mutation. ${ }^{30}$ Seven patients with the $\mathrm{D} 842 \mathrm{~V}$ mutation received treatment as compared with 14 patients with PDGFR $\alpha$ wild-type (WT) tumors. The 12-week clinical benefit rate (defined as stable disease, partial or complete response) was $50.0 \%(15.3-84.7)$ in patients with mutant tumors and $14.3 \%(2.6-38.5)$ in PDGFRa WT tumors. Median PFS was 32.1 weeks (5.0-35.9) in PDGFRa D842V-mutant tumors as compared with 6.1 weeks (5.7-6.3) in PDGFRa WT tumors. Median OS is yet to be reached for the patients with PDGFRa D842V GIST and is 24.9 weeks (14.4-49.1 in patients without PDGFRa mutations. The most common toxicities were fatigue $(38.1 \%)$, headache $(19 \%)$, nausea $(19 \%)$, peripheral edema (14.3\%) and infusion reaction (14.3\%). All patients who discontinued treatment within the study did so due to progressive disease. This limited study in a specific GIST population suggests some activity with olaratumab in PDGFRa D842V-mutant GIST, but further study is needed.

\section{Olaratumab in sarcomas: ongoing trials}

The Phase III double-blind placebo-controlled ANNOUNCE study of olaratumab and doxorubicin in patients with advanced or metastatic STS (NCT02451943) has completed

Table I Summary of ongoing and future trials with olaratumab in adults with STS

\begin{tabular}{llll}
\hline Phase & NCT & Drugs & Geographic location \\
\hline III & NCT0245I943 & Doxorubicin + olaratumab vs. doxorubicin + placebo & USA, Europe, Asia \\
I/II & NCT03283696 & Doxorubicin/ifosfamide + olaratumab & USA, Europe \\
I/II & NCT02659020 & Gemcitabine/docetaxel + olaratumab & USA, Europe \\
lb & NCT02783599 & Preoperative olaratumab + doxorubicin & USA, Europe \\
I & NCT03I2659I & Pembrolizumab + olaratumab & USA, Europe \\
\hline
\end{tabular}

Abbreviation: STS, soft tissue sarcoma. 
recruitment of 460 patients and analysis is awaited (Table 1). The first cycle of olaratumab was given at $20 \mathrm{mg} / \mathrm{kg}$ on Days 1 and 8 to ensure more rapid therapeutic serum olaratumab levels, given the data on drug exposure from the Phase II study. The two primary objectives of the study are the assessment of OS in all patients and in patients with leiomyosarcoma.

Further work to develop our knowledge of the mechanism of olaratumab will be carried out in a Phase Ib study of 40 patients with potentially resectable STS who will receive olaratumab alone at $20 \mathrm{mg} / \mathrm{kg}$ on Day 1 and Day 8 of a three-weekly schedule for the first cycle and then $20 \mathrm{mg} / \mathrm{kg}$ olaratumab alongside $75 \mathrm{mg} / \mathrm{m}^{2}$ doxorubicin on Day 1 every 21 days on cycle 2 (NCT02783599). From the third treatment cycle onward, patients will receive $15 \mathrm{mg} / \mathrm{kg}$ olaratumab on the same schedule alongside doxorubicin. Tissue samples will be taken pre and post olaratumab monotherapy with cycle 1, and tissue from later surgical resection will also be analyzed. The joint primary objectives of this study are to characterize circulating tumor cells pre and post olaratumab monotherapy and to analyze PDGFR $\alpha$, PDGFR $\beta$ and PDGF ligand expression changes. The study will also provide further efficacy, safety and pharmacokinetic data.

Gemcitabine and docetaxel have shown similar activity to doxorubicin in advanced STS, ${ }^{5}$ and there is a Phase Ib/II study of this combination with olaratumab in advanced or metastatic STS open for recruitment (NCT02659020). The primary objective of the Phase Ib part will be to recommend a Phase II dose of the triple combination. Both 15 and $20 \mathrm{mg} /$ $\mathrm{kg}$ olaratumab on Days 1 and 8 of a 21-day cycle will be evaluated, and 30 patients will be enrolled. The Phase II part of the study will aim to recruit 166 patients randomized 1:1 between gemcitabine, docetaxel and olaratumab vs. gemcitabine, docetaxel and placebo in a double-blinded fashion. The primary objective of the Phase II part of the trial is OS.

Another Phase I trial is evaluating the safety and tolerability of doxorubicin and ifosfamide in combination with olaratumab (NCT03283696). In addition, there is a Phase I trial of olaratumab in combination with pembrolizumab (NCT03126591) that is open for recruitment.

\section{Conclusion}

The outcome of adult patients with metastatic and locally advanced STS remains poor with a median OS of approximately 18 months. Previously, few systemic therapies have been available and largely limited to doxorubicin and ifosfamide. ${ }^{2}$ Over the past decade, a number of effective agents have emerged to treat STS, including gemcitabine/docetaxel, ${ }^{5}$ trabectedin, ${ }^{31}$ pazopanib $^{32}$ and eribulin. ${ }^{26}$
Despite extensive research, however, doxorubicin remains standard first-line therapy. The promising results of the randomized Phase II trial of olaratumab have led to rapid approval of this agent in Europe and the USA. Early studies have provided valuable information about the relationship between drug exposure and response. ${ }^{19,22,23}$ The randomized Phase III trial will define the role of this agent, and ongoing trials will provide valuable data regarding the activity of this drug with other therapeutics and better define the mechanism of action. Future studies should evaluate the potential role of olaratumab in bone tumors such as osteosarcoma and Ewing sarcoma as well as investigate further combinations.

The randomized Phase III trial in sarcomas has completed enrollment, and it is hoped that it will lead to another treatment option for patients with these challenging and heterogeneous diseases.

\section{Disclosure}

The authors report no conflicts of interest in this work.

\section{References}

1. Karavasilis V, Seddon BM, Ashley S, Al-Muderis O, Fisher C, Judson I. Significant clinical benefit of first-line palliative chemotherapy in advanced soft-tissue sarcoma. Cancer. 2008;112(7):1585-1591.

2. Judson I, Verweij J, Gelderblom H, et al. Doxorubicin alone versus intensified doxorubicin plus ifosfamide for first-line treatment of advanced or metastatic soft-tissue sarcoma: a randomised controlled phase 3 trial. Lancet Oncol. 2014;15(4):415-423.

3. Ryan CW, Merimsky O, Agulnik M, et al. PICASSO III: a phase III, placebo-controlled study of doxorubicin with or without palifosfamide in patients with metastatic soft tissue sarcoma. J Clin Oncol. 2016;34:3898-3905

4. Tap WD, Papai Z, Van Time BA, et al. Randomized phase 3, multicenter, open-label study comparing evofosfamide (Evo) in combination with doxorubicin (D) vs. D alone in patients (pts) with locally advanced, unresectable or metastatic soft tissue sarcoma. Ann Oncol. 2016;27:483-492.

5. Seddon B, Strauss SJ, Whelan J, Leahy M, Woll PJ. Gemcitabine and docetaxel versus doxorubicin as first-line treatment in previously untreated advanced unresectable or metastatic soft-tissue sarcomas. The Lancet Oncology. 2017;18(10):1397-1410.

6. Carvalho I, Milanezi F, Martins A, Reis RM, Schmitt F. Overexpression of platelet-derived growth factor receptor alpha in breast cancer is associated with tumour progression. Breast Cancer Res. 2005;7: R788-R795.

7. Ramos AH, Dutt A, Mermel C, et al. Amplification of chromosomal segment 4q12 in non-small cell lung cancer. Cancer Biol Ther. 2009;8(21): 2042-2050.

8. Cancer Genome Atlas Research Network Comprehensive genomic characterization defines human glioblastoma genes and core pathways. Nature. 2008;455:1061-1068.

9. Dolloff NG, Shulby SS, Nelson AV, et al. Bone-metastatic potential of human prostate cancer cells correlates with Akt/PKB activation by alpha platelet-derived growth factor receptor. Oncogene. 2005;24(45): 6848-6854.

10. Adams SF, Hickson JA, Hutto JY, Montag AG, Lengyel E, Yamada SD. PDGFR-alpha as a potential therapeutic target in uterine sarcomas. Gynecol Oncol. 2007;104(3):524-528. 
11. Shah GD, Loizos N, Youssoufian H, Schwartz JD, Rowinsky EK. Rationale for the development of IMC-3G3, a fully human immunoglobulin G subclass 1 monoclonal antibody targeting the platelet-derived growth factor receptor alpha. Cancer. 2010;116:1018-1026.

12. Li C, Wang Y, Lu S, et al. MiR-34a inhibits colon cancer proliferation and metastasis by inhibiting platelet-derived growth factor receptor $\alpha$. Mol Med Rep. 2015;12(5):7072-7078.

13. Zhang Z, Ren X, Lu X, et al. GZD856, a novel potent PDGFR $\alpha / \beta$ inhibitor, suppresses the growth and migration of lung cancer cells in vitro and in vivo. Cancer Lett. 2016;375(1):172-178.

14. Liu K-W, Hu B, Cheng S-Y. Platelet-derived growth factor receptor alpha in glioma: a bad seed. Chin J Cancer. 2011;30:590-602.

15. Skobe M, Fusenig NE. Tumorigenic conversion of immortal human keratinocytes through stromal cell activation. Proc Natl Acad Sci USA. 1998;95(3):1050-1055.

16. Cao Y. Multifarious functions of PDGFs and PDGFRs in tumor growth and metastasis. Trends Mol Med. 2013;19:460-473.

17. Loizos N, Xu Y, Huber J, et al. Targeting the platelet-derived growth factor receptor alpha with a neutralizing human monoclonal antibody inhibits the growth of tumor xenografts: implications as a potential therapeutic target. Mol Cancer Ther. 2005;4(3):369-379.

18. Jayson GC, Parker GJ, Mullamitha S, et al. Blockade of platelet-derived growth factor receptor-beta by CDP860, a humanized, PEGylated diFab', leads to fluid accumulation and is associated with increased tumor vascularized volume. J Clin Oncol. 2005;23(5):973-981.

19. Doi T, Ma Y, Dontabhaktuni A, Nippgen C, Nippgen J, Ohtsu A. Phase I study of olaratumab in Japanese patients with advanced solid tumors. Cancer Sci. 2014;105(7):862-869.

20. Gerber DE, Gupta P, Dellinger MT, et al. Stromal platelet-derived growth factor receptor a (PDGFR $\alpha$ ) provides a therapeutic target independent of tumor cell PDGFR $\alpha$ expression in lung cancer xenografts. Mol Cancer Ther. 2012;11(11):2473-2482.

21. Russell MR, Liu Q, Fatatis A. Targeting the \{alpha\} receptor for platelet-derived growth factor as a primary or combination therapy in a preclinical model of prostate cancer skeletal metastasis. Clin Cancer Res. 2010;16(20):5002-5010.

22. Chiorean EG, Sweeney C, Youssoufian H, et al. A phase I study of olaratumab, an anti-platelet-derived growth factor receptor alpha (PDGFR $\alpha$ ) monoclonal antibody, in patients with advanced solid tumors. Cancer Chemother Pharmacol. 2014;73(3):595-604.
23. Tap WD, Jones RL, Van Tine BA, et al. Olaratumab and doxorubicin versus doxorubicin alone for treatment of soft-tissue sarcoma: an open-label phase $\mathrm{lb}$ and randomised phase 2 trial. Lancet. 2016; 388(10043):488-497.

24. Jones RL. Utility of dexrazoxane for the reduction of anthracyclineinduced cardiotoxicity. Expert Rev Cardiovasc Ther. 2008;6: 1311-1317.

25. Ebos JML, Kerbel RS. Antiangiogenic therapy: impact on invasion, disease progression, and metastasis. Nat Rev Clin Oncol. 2011;8(4):210-221.

26. Schöffski P, Chawla S, Maki RG, et al. Eribulin versus dacarbazine in previously treated patients with advanced liposarcoma or leiomyosarcoma: a randomised, open-label, multicentre, phase 3 trial. Lancet. 2016;387(10028):1629-1637.

27. Shirley M. Olaratumab: first global approval. Drugs. 2017;77(1): $107-112$.

28. Gerber DE, Campbell TC, Swanson P, et al. A randomized phase 2 study of a human antiplatelet-derived growth factor a (PDGFR $\alpha$ ) monoclonal antibody (olaratumab, IMC-3G3) with paclitaxel/carboplatin or paclitaxel/carboplatin alone in previously untreated patients with advanced non-small cell lung cancer (NSCLC). J Clin Oncol. 2014;32 (15 suppl):8050-8050.

29. Cassier PA, Fumagalli E, Rutkowski P, et al; European Organisation for Research and Treatment of Cancer. Outcome of patients with platelet-derived growth factor receptor alpha-mutated gastrointestinal stromal tumors in the tyrosine kinase inhibitor era. Clin Cancer Res. 2012;18:4458-4464.

30. Wagner AJ, Kindler H, Gelderblom H, et al. A phase II study of a human anti-PDGFR $\alpha$ monoclonal antibody (olaratumab, IMC-3G3) in previously treated patients with metastatic gastrointestinal stromal tumors. Ann Oncol. 2017;28(3):541-546.

31. Demetri GD, von Mehren M, Jones RL, et al. Efficacy and safety of trabectedin or dacarbazine for metastatic liposarcoma or leiomyosarcoma after failure of conventional chemotherapy: results of a phase III randomized multicenter clinical trial. J Clin Oncol. 2016;34(8): 786-793.

32. van der Graaf WT, Blay JY, Chawla SP, et al; EORTC Soft Tissue and Bone Sarcoma Group, PALETTE Study Group. Pazopanib for metastatic soft-tissue sarcoma (PALETTE): a randomised, double-blind, placebocontrolled phase 3 trial. Lancet. 2012;379:1879-1886.
Clinical Pharmacology: Advances and Applications

Publish your work in this journal

Clinical Pharmacology: Advances and Applications is an international, peer-reviewed, open access journal publishing original research, reports, reviews and commentaries on all areas of drug experience in humans. The manuscript management system is completely online and includes a very quick and fair peer-review system, which is all easy to use.

\section{Dovepress}

Visit http://www.dovepress.com/testimonials.php to read real quotes from published authors. 